\title{
Effect of Screw Configuration on the Dispersion and Properties of Polypropylene/Multiwalled Carbon Nanotube Composite
}

\author{
Gulstan S. Ezat ${ }^{1 *}$, Adrian L. Kelly ${ }^{2}$, Mansour Youseffi ${ }^{3}$, Phil D. Coates ${ }^{2}$ \\ ${ }^{1}$ Department of Physics, College of Science, University of Sulaimani, Qlyasan Road, Sulaimani 4600, Iraq \\ ${ }^{2}$ Polymer IRC, Faculty of Engineering and Informatics, University of Bradford, Bradford, BD7 1DP, UK \\ ${ }^{3}$ Faculty of Engineering and Informatics, University of Bradford, Bradford, BD7 1DP, UK
}

\begin{abstract}
The effect of extruder screw configuration on the dispersion and properties of compatibilised polypropylene (PP)/Multi-walled carbon nanotube (MCNT) composite is investigated. Three principle screw designs with mainly conveying elements (medium intensity), kneading elements (high intensity) and folding elements (chaotic mixing) were used to prepare polypropylene nanocomposites containing $4 \mathrm{wt} \%$ of maleic anhydride grafted polypropylene (MAH-g-PP) compatibilizer and different nanotube loadings. The effect of each screw configuration and nanotube loading on the tensile, rheological and electrical properties of the nanocomposites were studied. The screw configurations were found to have a strong influence on the electrical resistivity whilst only slightly affected the tensile properties of the nanocomposites. Scanning electron microscopy examinations showed that the use of screw configuration consisting of kneading elements promoted the dispersion of nanotubes and resulted in a low electrical percolation at $2 \mathrm{wt} \%$ of MCNT.
\end{abstract}

\footnotetext{
*Correspounding arthur email address gulstan.ezat@univsul.edu.iq
} 


\section{Introduction}

In preparation of carbon nanotube polymer composites, effective mixing of raw materials is a critical step of the process. The final characteristics of the product are strongly dependent upon the uniformity of the mixture; non-uniform dispersion of nanotubes within the polymer can cause a significant variation in mechanical and electrical properties of the nanocomposite [1-4]. However agglomeration of nanotubes during production of nanocomposites is inevitable due to the strong Van der Waals interaction between individual nanotubes and the high surface energy associated with these nano-sized fillers. Dispersion mechanisms occur by wetting of the primary agglomerates with polymer melt, penetration of polymer chains into the agglomerates and then breaking them into individual nanotubes by rupture and erosion. These mechanisms occur during compounding of the nanocomposites and are sensitive to the characteristics of the polymer and nanotubes in addition to the processing equipment utilized during processing [5].

Polypropylene (PP) is one of the most widely used thermoplastics in the world, due to its low cost, low density, high thermal stability and resistance to corrosion. Blending polypropylene with carbon nanotubes to form nanocomposites is an approach applied to further increase its potential use in the automotive, packaging and electronic industries. Suggested applications of polypropylene carbon nanotube composites have included strain sensing [6], electrostatic dissipation [7] and electromagnetic interference shielding [8].

Different approaches have been proposed to prepare PP/CNT include melt mixing $[9,10]$, insitu polymerization [11,12] and solution [13,14] methods. Synthesis methods can directly influence nanotube dimension, dispersion and interaction with the polymer. Compared to other methods melt compounding is regarded as the easiest and most environmentally friendly method 
for incorporating carbon nanotubes into thermoplastic polymers. It involves a combination of traditional industrial processes such as extrusion and injection molding which are suitable for mass production of nanocomposites $[15,16]$. In addition, application of the high shear used during compounding may decrease the tendency of nanotubes to agglomerate.

Twin screw extrusion is regarded as the preferred melt compounding method to produce polymer nanocomposites $[17,18]$. The modular nature of most twin screw-extruder screws gives the process an inherent flexibility to tailor mixing intensity and residence time. The barrel and screw elements of twin screw-extruder can be replaced and rearranged to achieve optimum mixing characteristics. The most popular screw elements for modular co-rotating twin screw extruders are conveying elements, kneading elements and mixing elements. Conveying elements (also known as feeding elements) are used to direct and transfer the material toward the die, whilst the reversible conveying elements are used to retain the material in the screw channels and form a melt seal. The main function of kneading elements is for plastification of polymers, dispersion of filler and enhancement of the mixing process. Mixing elements are used in certain applications when the desired mixing is not achieved by kneading elements [15]. Each element of the extruders can have dramatic influence on the homogeneity and property of the final products.

Within the literature, the influence of carbon nanotube functionalization $[19,20]$ and the use of different compatibilising agents $[10,21]$ to enhance dispersion and increase the compatibility of carbon nanotubes and polypropylene have been extensively studied. However the effect of screw configuration has not been sufficiently examined because of the complex correlation between processing parameters and structure of these nanocomposites. Furthermore, the few studies which have investigated the effect of screw configuration have been carried out at constant 
nanotube loading and have concentrated on specific characteristics of PP/CNT nanocomposites $[22,23]$. Investigation of the effect of screw configuration on the dispersion of MCNT in PP [22] and poly(lactic acid) PLA [24] matrices concluded that a combination of high screw rotation speed and a screw configuration mainly consisting of mixing elements were most effective to disperse nanotubes within the polymer. Further changes to the screw configuration were found to have insignificant effect on the resistivity of the nanocomposites [22]. It has also been observed that the utilization of a screw with long residence time was not necessary to achieve uniform dispersion of nanotubes [25] while the choice of the type of screw elements was critical in dispersing nanotubes within the polymer [23].

The aim of this study was to quantify the effect of extruder screw configuration on the properties of melt compounded polypropylene/multiwalled carbon nanotube composites (PP/MCNT) to investigate the effect on dispersion and properties of PP/MCNT composite at different nanotube

loadings. Importantly, the study reports a comprehensive investigation of the relationship between screw configuration and mechanical, rheological and electrical properties of PP/MCNT at various nanotubes loading, which has not previously been reported.

\section{Materials and methodology}

\subsection{Materials}

Commercially available acid purified MCNT with $95 \mathrm{wt} \%$ purity were used in this study, obtained from Cheap Tubes Inc. (USA). The MCNT were synthesized by catalytic vapor deposition method and had a diameter of 20-40 $\mathrm{nm}$ and length of 10-30 $\mu \mathrm{m}$. Polypropylene homopolymer (Innovene 100-GAO3) with a melting point of $163^{\circ} \mathrm{C}$ and melt 
flow rate of $3.0 \mathrm{~g} / 10 \mathrm{~min}\left(230^{\circ} \mathrm{C}, 2.16 \mathrm{~kg}\right)$ was selected as the polymer matrix. In order to optimize the potential for effective mixing, polypropylene supplied in the form of pellets was cryogenically ground into coarse powder of diameter of approximately $100 \mu \mathrm{m}$ by Queen's University, Belfast. In order to increase compatibility between PP and MCNT, polypropylene grafted maleic anhydride (MA2) containing 10wt $\%$ of maleic anhydride supplied in powder form by Sigma Aldrich, was used as compatibilizer.

\subsection{Preparation of PP/MCNT composites}

Composites of PP/MCNT were prepared at $0,2,4,6,8,10$ and $12 \mathrm{wt} \%$ MCNT loading and with $4 \mathrm{wt} \% \mathrm{MA} 2$ compatibilizer. To achieve a homogenous mixture, prior to extrusion the PP, MCNT and MA2 powder were tumble blended together for 10 minutes using a Turbula mechanical mixer. Each mixture was melt compounded using an intermeshing co-rotating twin screw extruder (PRISM-TSE-16-TC) having a screw diameter of $16 \mathrm{~mm}$ and a length to diameter (L/D) ratio of 15:1. Compounding was performed at a screw speed of $100 \mathrm{rev} \mathrm{min}^{-1}$ and set barrel temperatures of $180-200-210^{\circ} \mathrm{C}$ (from feed to die), respectively. A single screw feeder designed at the University of Bradford was used to feed the powdered mixtures; due to torque limitations different feeding rates were used for each screw configuration. The extruded nanocomposite strand was quenched in water and pelletized for subsequent forming and analysis. For comparision purpose extruded polypropylene (EPP) was also processed.

\subsection{Characteristic of Screw Configurations}

In addition to the standard screw configuration (SC1) supplied by Thermo PRISM, two different screw configurations ( $\mathrm{SC} 2$ and $\mathrm{SC} 3$ ) were designed and used to prepare $\mathrm{PP} / \mathrm{MCNT}$ composites. The designs of three screw configurations are shown in Figure 1. The barrel of the 
twin screw extruder consists of four zones which are the feeding, melting, mixing and metering sections. The screws were designed by modifying the type and position of screw elements in the melting and mixing region, while the design of feeding and metering section for all screw configurations were the same. The standard screw configuration SC1 consisted of forward conveying elements in the melting section followed by a set of kneading block elements in the mixing section. The high intensity screw configuration $\mathrm{SC} 2$ was based on $\mathrm{SC} 1$ with the addition of another set of kneading block elements in the mixing section. Besides the kneading elements a reverse conveying element was added after the mixing section, with the aim of increasing the degree of filling and the residence time of SC2 and consequently improves dispersion. Screw configuration SC3 was designed to establish chaotic mixing characteristics. Folding elements were used in this configuration to increase the pressure and the residence time in the melting and mixing zone. The individual screw elements like conveying, kneading, folding and metering elements used in designing of the screw configuration (SC1-SC3) are shown in Figure 2. 

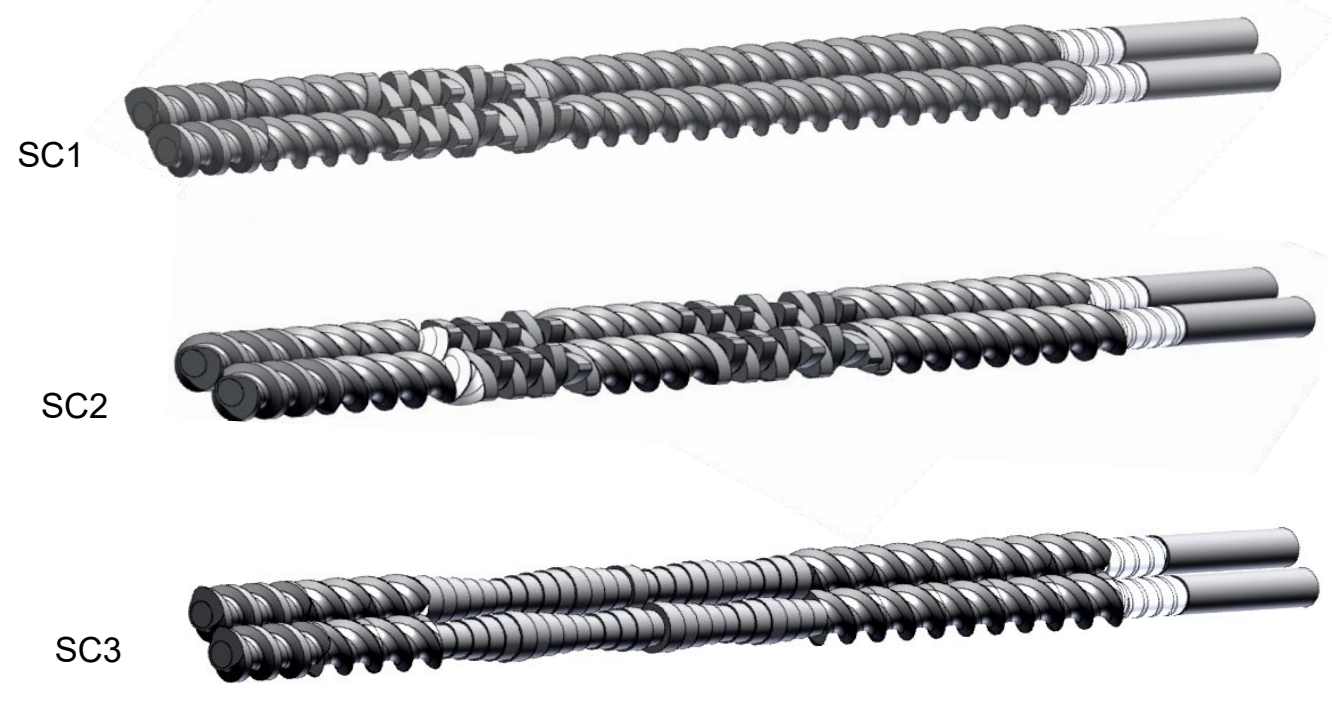

Figure 1 Schematic representation of the geometry of screw configurations SC1, SC2 and SC3

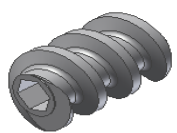

Metering elements

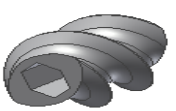

Conveying elements

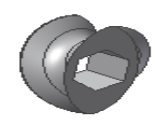

Half conveying elements
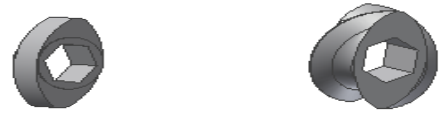

Kneading elements Reversing elements

Folding elements

Figure 2 Diagrams of individual screw elements for twin screw extruder 


\subsection{Sample Preparation}

Tensile test specimens with neck dimensions of $33 \times 6 \times 2 \mathrm{~mm}$ were prepared using a pneumatic ram injection molding machine at a set temperature of $230^{\circ} \mathrm{C}$ and an injection pressure of 0.35 MPa. For electrical measurements sheets of film were prepared by a heated hydraulic press (Moore, UK). A mass of $54 \mathrm{~g}$ of extruded pellets was pressed between a pair of polished metal platens at a temperature of $230{ }^{\circ} \mathrm{C}$ to achieve a compression molded sheet with $1 \mathrm{~mm}$ thickness. Samples were initially preheated at $50 \mathrm{MPa}$ for $120 \mathrm{~s}$ and then molded under $300 \mathrm{MPa}$ for $90 \mathrm{~s}$. The molded films were quenched in cold water and used for electrical measurements.

\section{Characterization of PP/MCNT Composite}

\subsection{Tensile Properties}

Tensile tests were carried out at room temperature using an Instron 5564 tensometer at a deformation rate of $5 \mathrm{~mm} / \mathrm{min}$ according to BS EN ISO 527-1. From each measurement five specimens were tested and the average values along with the corresponding mean value of standard errors calculated.

\subsection{Rheological Properties}

Rheological measurement was performed using a parallel plate rotational rheometer (Anton Paar MCR 501) in dynamic oscillation mode. The test was conducted with parallel plates of $25 \mathrm{~mm}$ diameter at a constant gap of $1 \mathrm{~mm}$. Frequency sweeps between $10^{-2}-10^{2} \mathrm{~Hz}$ at $5 \%$ strain were applied at $200^{\circ} \mathrm{C}$ within the linear viscoelastic range of the materials. 


\subsection{Electrical Resistivity}

Volume resistivity of the nanocomposites was measured by a two-probe technique using a Keithley 6517A electrometer/high resistance meter and 8009 resistivity fixture device. Square shaped specimens with dimensions of $100 \times 100 \times 1 \mathrm{~mm}^{3}$ were cut from the compression molded sheets and tested according to ASTM D-257. The measurement was performed by applying a constant voltage of $50 \mathrm{~V}$ across the sample and measuring the resultant current passing through the sample. All the samples were tested at room temperature and the values of volume resistivity ( $\rho v)$ automatically calculated by the 6517A high resistance software.

\subsection{Characterization of MCNT Dispersion}

Scanning electron microscopy (ESEM, FEI Quanta 400) of fractured surfaces was used to examine the morphology of PP/MCNT composites prepared by the three screw configurations. Tensile specimens were fractured in liquid nitrogen and characterized at accelerating voltage $20 \mathrm{KV}$. In order to prevent electron charging the specimens were coated with a thin layer of gold.

\subsection{Residence Time Distribution Measurements}

Residence time distribution (RTD) of the three screw configurations (SC1-SC3) were measured by a UV-fluorescence spectroscopy technique. Polypropylene powder was used as the main feedstock material and Uvitex-optical brightener (Uvitex/PP master batch $30 \mathrm{wt} \%$ ) used as a tracer. A high temperature fibre optic probe (Ocean Optics USB 2000) was fixed in the extruder die. A pellet of the tracer was fed into the extruder hopper after steady state conditions were reached and the fluorescent intensity of the tracer recorded every 0.5 seconds. The intensity of 
the emitted spectrum was monitored by Labview software. The mean residence time ( $\left.\mathrm{t}^{\prime}\right)$ was calculated by the following equation [26].

$$
t^{\prime}=\frac{\sum_{0}^{\infty} t \cdot C(t) \cdot \Delta t}{\sum_{0}^{\infty} C(t) \cdot \Delta t}
$$

Here $\mathrm{C}(\mathrm{t})$ is the intensity of the tracer at time $\mathrm{t}$ and $\Delta \mathrm{t}$ is time increase.

\section{Results and discussion}

\subsection{Tensile Properties}

Figure 3 shows the tensile modulus of compatibilised polypropylene as a function of carbon nanotube content for screw configurations SC1, SC2 and SC3. From Figure 3, it can be seen that the tensile modulus of compatibilised polypropylene and nanocomposite was only slightly affected by screw configuration. The modulus of all the blends (PP/4wt\%MA2) increased with increase carbon nanotube content. This increase was more pronounced for the nanocomposite prepared by the medium intensity screw configuration (SC1). At $12 \%$ of carbon nanotube loading the modulus of nanocomposites prepared by SC1, SC2 and SC3 increased by $80 \%, 65 \%$ and $54 \%$ respectively. The smaller increase in the tensile modulus of nanocomposites prepared by SC2 and SC3 screw configurations is most likely to be due to the breakage of carbon nanotube length by the intensive shear produced by high intensity (SC2) and chaotic (SC3) screw configurations [27,28] which decreased the benefit of their large aspect ratio in the polymer [29].

Corresponding tensile strength results are illustrated in Figure 4. It can be observed that the configurations with higher mixing intensity produced an adverse effect on tensile strength of compatibilised polypropylene and the value of tensile strength for the blend prepared by SC3 was found to be the lowest, possibly due to the degradation of MA2 compatibilizer as a 
result of the longer residence time for $\mathrm{SC} 2$ and $\mathrm{SC} 3$ screw configurations. The tensile strength of all the blends was improved by addition of carbon nanotubes and the greatest enhancement observed for the nanocomposite prepared by $\mathrm{SC} 1$ configuration. However, the overall effect of carbon nanotubes on tensile strength was relatively minor, with enhancement of up to $9 \%$ above that of neat polypropylene observed.

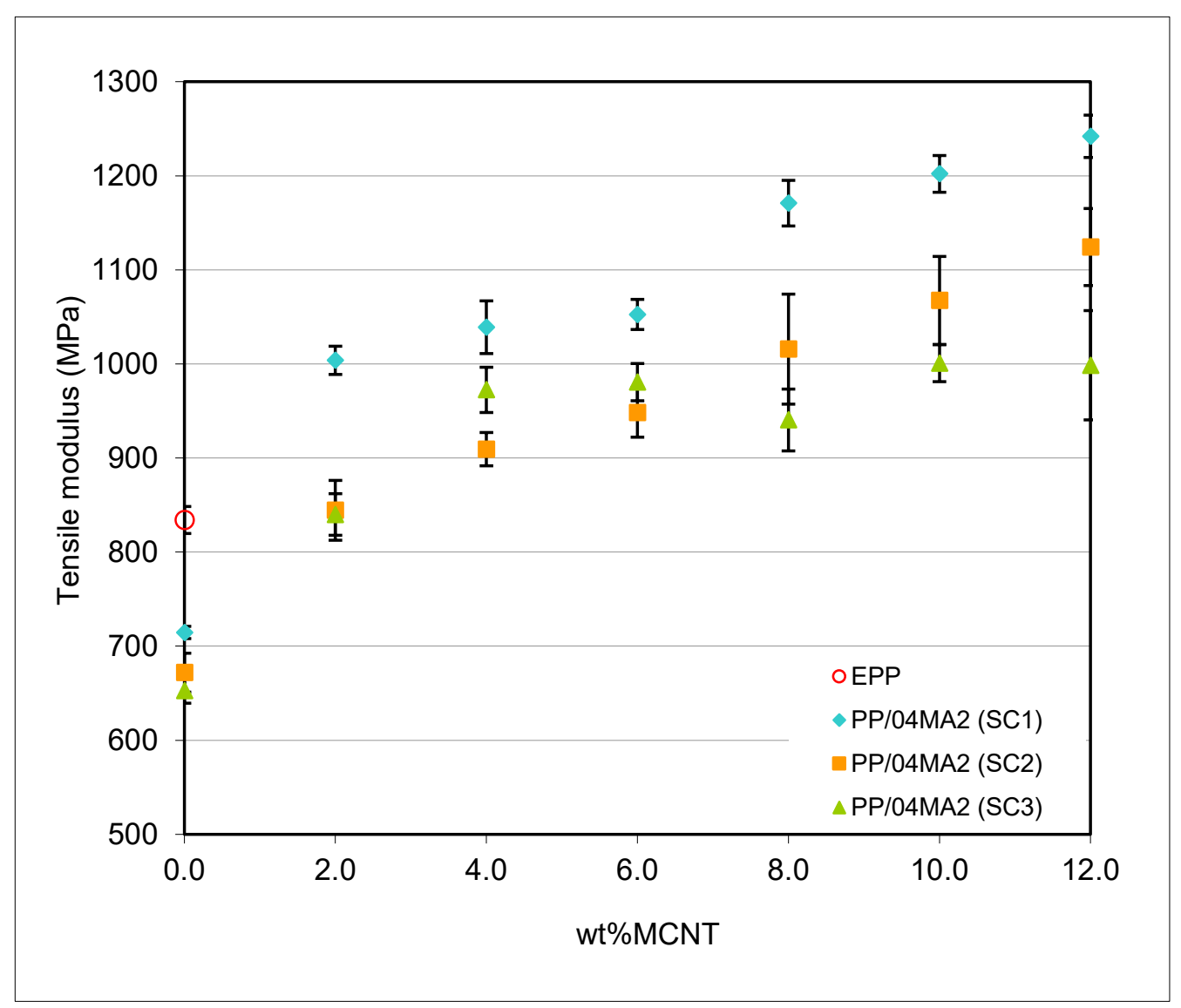

Figure 3 Tensile modulus of compatibilised polypropylene at different MCNT loadings prepared by screw configurations $\mathrm{SC} 1, \mathrm{SC} 2$, and $\mathrm{SC} 3$ 


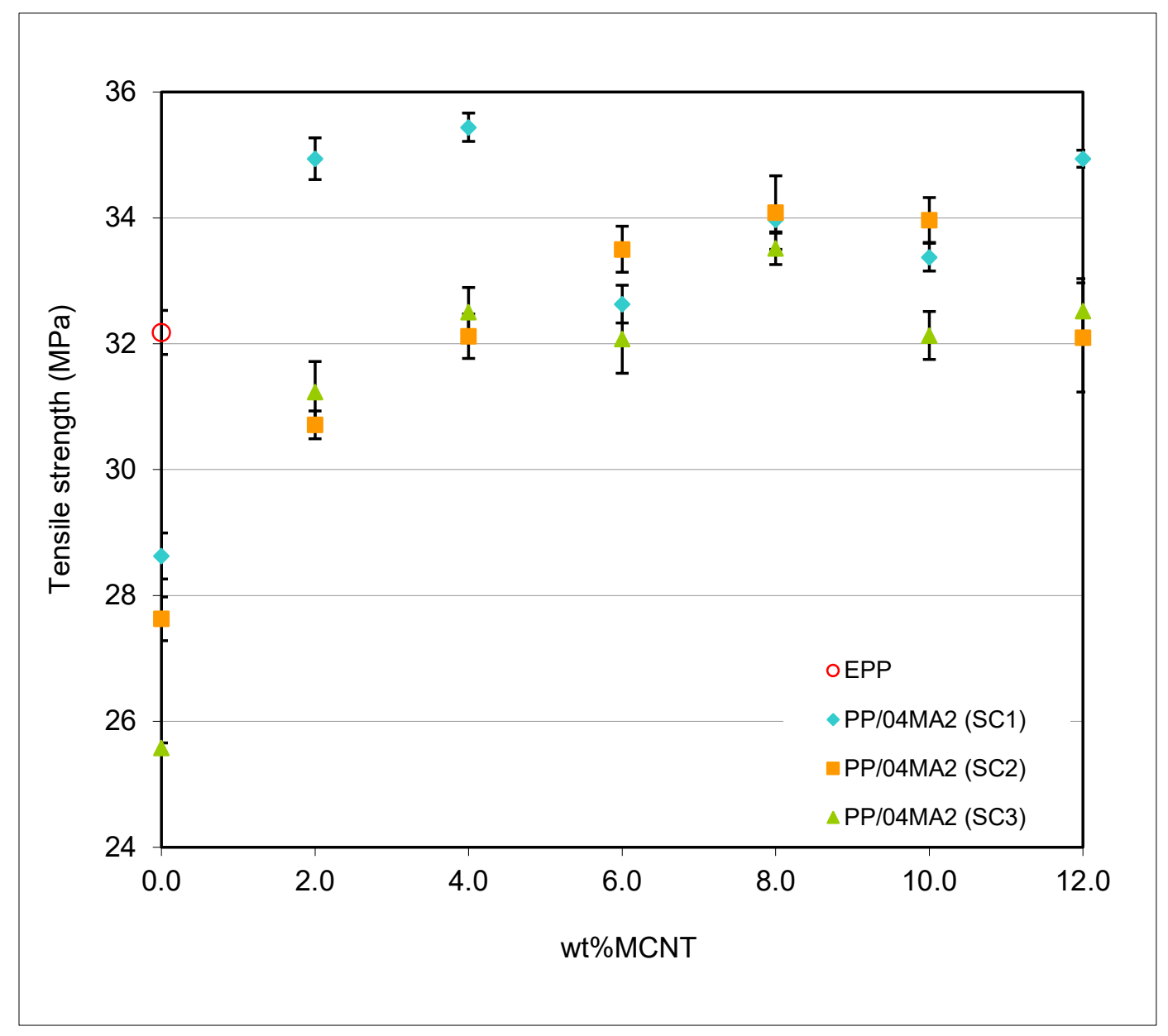

Figure 4 Tensile strength of compatibilised polypropylene at different MCNT loadings prepared by screw configurations $\mathrm{SC} 1, \mathrm{SC} 2$, and $\mathrm{SC} 3$

\subsection{Rheological properties}

Figure 5 represents damping factor (ratio between loss and storage modulus) obtained from rheological measurements for $\mathrm{PP} / 4 \mathrm{wt} \% \mathrm{MA}$ containing 2 and $4 \mathrm{wt} \% \mathrm{MCNT}$ loading prepared by the three screw configurations. The decrease in the slope of damping factor at low frequencies has been proposed to indicate formation of interconnected nanotube networks in the nanocomposite and transition from liquid-like to solid-like behavior [30-33]. As the value of damping factor presents the ratio between viscous and elastic properties of the materials, a high value of damping factor indicates predominantly viscous behavior of the materials while a low value relates to elastic behavior. 
From Figure 5 it can be seen that application of different screw configuration had insignificant effect on the damping behavior of compatibilised polypropylene and nanocomposites. The damping behavior of compatibilised polypropylene prepared by different screw configurations was found to be dependent on frequency and showed predominantly viscous characteristics. For all the blends addition of nanotubes decreased the damping factor and the curve became flatter with increasing nanotube loading. This indicates an increase in the elastic response of the material and is evidence of the restriction of mobility of polymer chains in the presence of rheological percolation at 2wt $\%$ MCNT.

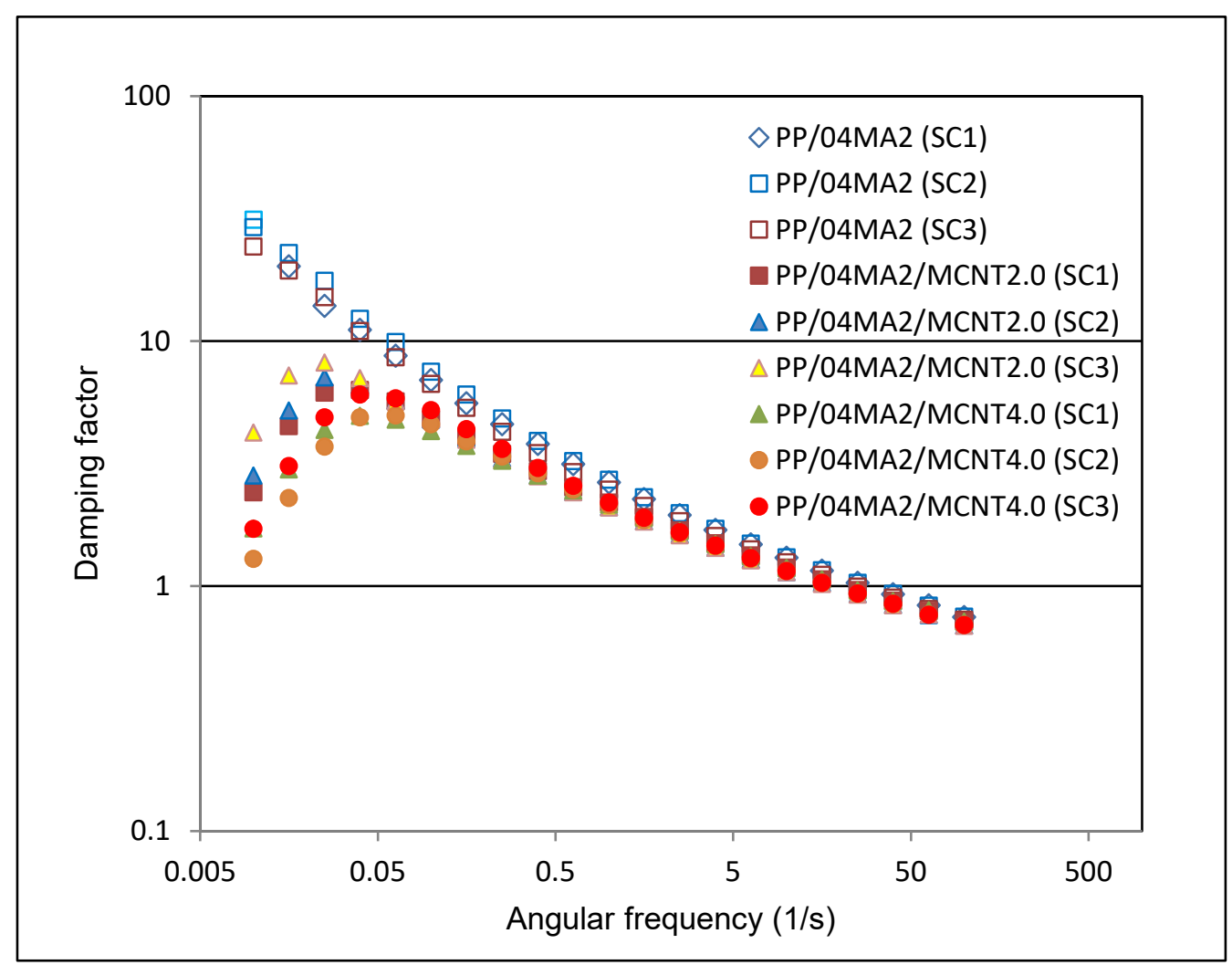

Figure 5 Damping factor of compatibilised polypropylene containing 2 and $4 \mathrm{wt} \% \mathrm{MCNT}$ loading prepared by screw configurations $\mathrm{SC} 1, \mathrm{SC} 2$ and $\mathrm{SC} 3$ 


\subsection{Electrical Resistivity}

Figure 6 shows the volume resistivity of compatibilised polypropylene as a function of nanotube content for SC1, SC2 and SC3 screw configurations. A clear dependence of the electrical resistivity on the type of screw configuration was found. For all screw configurations, the electrical resistivity decreased with increasing nanotube loading. The rapid drop in the electrical resistivity of the nanocomposites prepared by $\mathrm{SC} 1$ and $\mathrm{SC} 3$ at $8 \mathrm{wt} \%$ of nanotube content indicates the formation of electrical percolation. Above $8 \mathrm{wt} \%$ of nanotubes the resistivity of both nanocomposites decreased gradually and demonstrated a plateau with the lowest value being observed for nanocomposite prepared by SC1. The lowest percolation threshold was observed at $4 \mathrm{wt} \%$ of nanotube content for the nanocomposite prepared by SC2. Above $4 \mathrm{wt} \%$ of nanotube content the resistivity of the nanocomposite prepared by SC2 showed a step like drop up to $8 \mathrm{wt} \%$ of nanotube contents. Similar behavior has also been observed for PP/MCNT composites [34] and this was explained by the concept of a double percolation threshold. It was reported that due to the semi-crystalline structure of the polypropylene matrix, carbon nanotubes tend to be present in the amorphous phase between the lamellae rather than in the crystal phase and consequently, different types of conduction networks are formed in each phase. Another possible reason for this phenomenon can be due to the high shear intensity produced by SC2 configuration which resulted in a more uniform dispersion of carbon nanotubes within the nanocomposite and consequently helped the network formation at lower nanotube contents.

To estimate an accurate electrical percolation in the nanocomposite prepared with three screw configurations, the experimental values of resistivity were fitted to the power law relationship of classical percolation theory described in equation 2 [35-37]: 


$$
\sigma_{\mathrm{dc}} \sim(\Phi-\Phi \mathrm{c})^{\mathrm{t}} \quad \text { for } \Phi>\Phi \mathrm{c}
$$

Here $\sigma_{\mathrm{dc}}$ is the conductivity of the nanocomposite and it is equal to the inverse of resistivity, $\Phi$ is the concentration of $\mathrm{MCNT}$, $\Phi \mathrm{c}$ is the percolation concentration and $\mathrm{t}$ is the conductivity exponent. According to percolation theory the conductivity exponent $(\mathrm{t})$ represents the average number of contacts per particle at the percolation threshold [35]. For three dimensional systems the theoretical value of $t$ is around 2 .

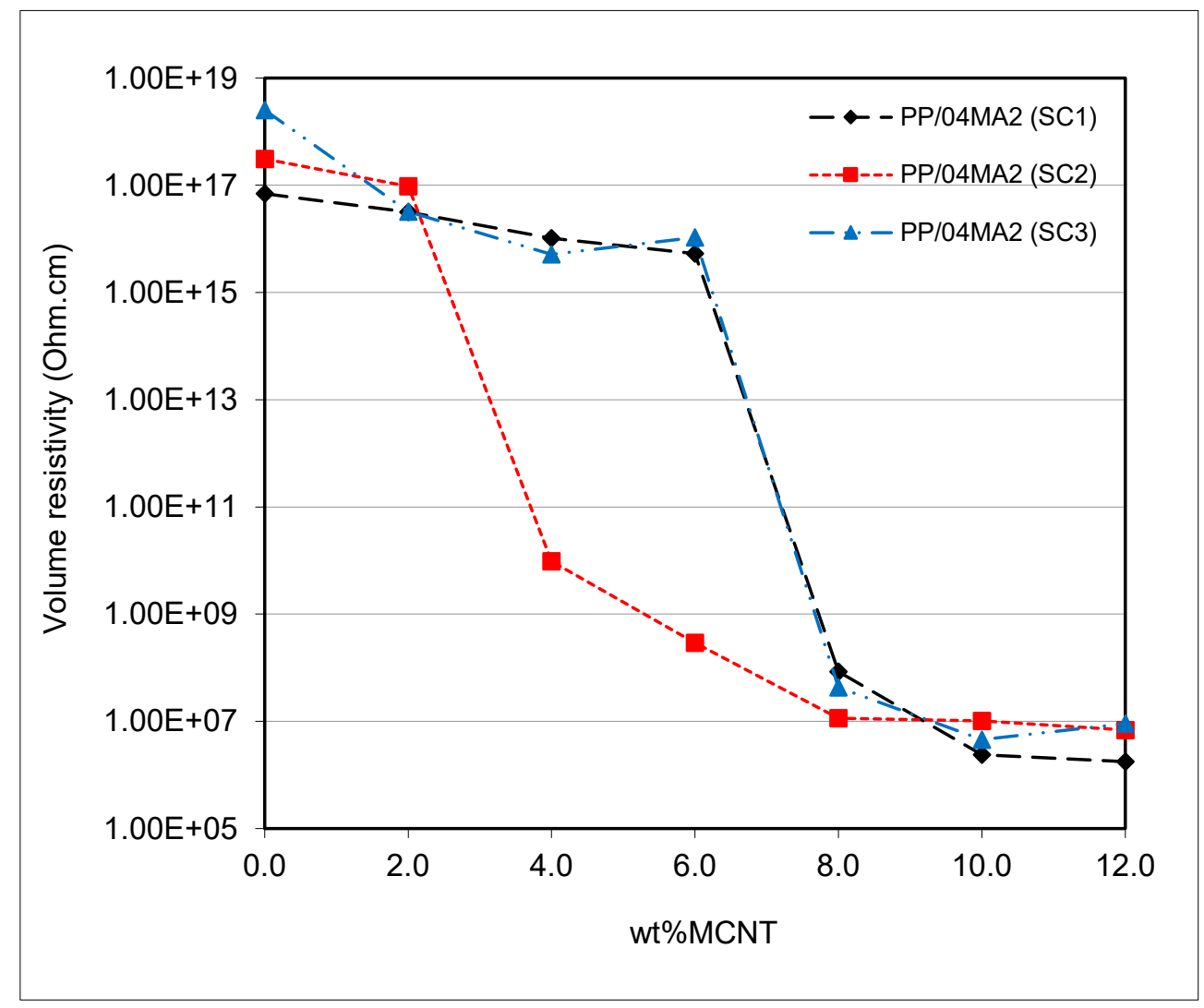

Figure 6 Volume resistivity of compatibilised polypropylene at different MCNT loadings for screw configurations SC1, SC2 and SC3.

Figure 7 shows a log-log plot of conductivity versus (Ф-Фc) for the nanocomposite prepared by $\mathrm{SC} 1, \mathrm{SC} 2$ and SC3. The value of $\mathrm{t}$ was found by changing the values of $\Phi \mathrm{c}$ until the best linear 
fit to the experimental data obtained. The parameters obtained by fitting the experimental data to equation 2 are shown in Table 1.

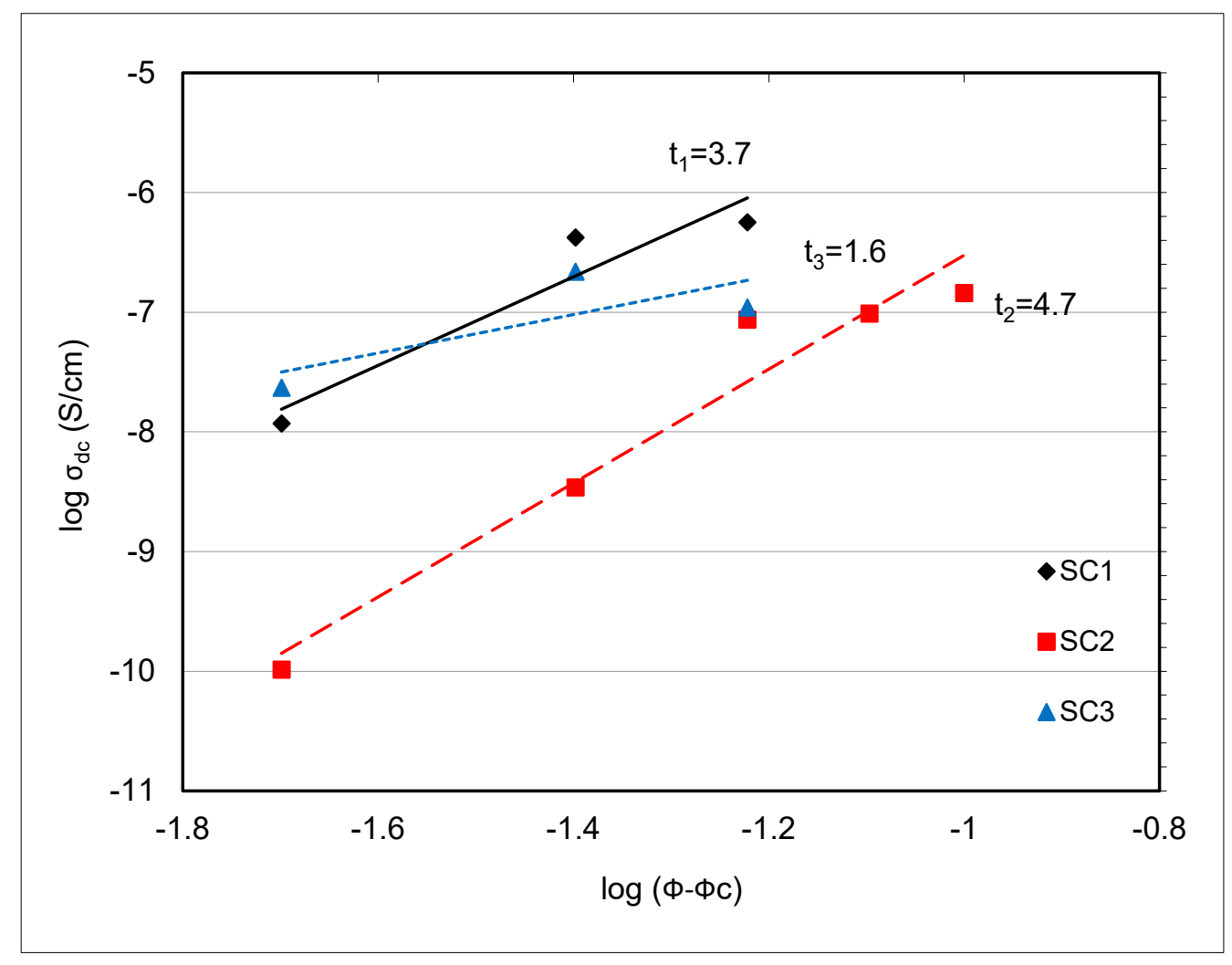

Figure 7 Logarithmic plot of conductivity versus $(\Phi-\Phi c)$ for nanocomposites prepared by screw configurations $\mathrm{SC} 1, \mathrm{SC} 2$, and $\mathrm{SC} 3$.

The results shown in Table 1 demonstrate that the values of critical exponent ( $t$ ) are 3.7, 4.7 and 1.6 for SC1, SC2 and SC3 configurations respectively. Also the values of percolation threshold were calculated at $6 \%, 2 \%$ and $6 \%$ of nanotube loadings for the screw configurations $\mathrm{SC} 1, \mathrm{SC} 2$ and $\mathrm{SC} 3$ respectively. The deviation in the value of $\mathrm{t}$ for the nanocomposite prepared by $\mathrm{SC} 1$ and $\mathrm{SC} 2$ can indicate a greater tunneling barrier between carbon nanotubes $[35,38]$ and is probably 
due to the fact that the theoretical value of $t$ is predicted for a system where the network is formed by particles which are in physical contact, while in CNT/Polymer composites the nanotubes are separated by an insulating layer of polymer and charge transport occurs through a tunneling process [39]. Deviations in experimental values of $t$ have also been reported by other studies and were explained by non-identical properties of carbon nanotubes (such as length, diameter, chirality, and entanglements) and insufficient contact between them [40,41].

\begin{tabular}{|c|c|c|}
\hline screw configurations & $t$ & $\Phi \mathrm{c} / \mathrm{wt}$ \\
\hline $\mathrm{SC} 1$ & 3.7 & 6.0 \\
\hline $\mathrm{SC} 2$ & 4.7 & 2.0 \\
\hline $\mathrm{SC} 3$ & 1.6 & 6.0 \\
\hline
\end{tabular}

Table 1 Scaling law parameters for the nanocomposite prepared by SC1, SC2 and SC3 screw configurations

According to equation 3 [35,39], tunneling between nanotubes separated by a thin layer of polymer should obey the following relationship between DC conductivity $\left(\sigma_{\mathrm{dc}}\right)$ and filler concentration $(\varnothing)$ :

$$
\ln \sigma_{\mathrm{dc}} \propto-\varnothing^{-1 / 3}
$$

The linear relationship between conductivity and filler concentration has been suggested to predict the tunneling mechanism in CNT/polymer composite [35, 42]. Figure 8 shows that the linear behavior was only observed for the nanocomposite prepared with screw configuration SC2 below $8 \mathrm{wt} \%$ of nanotube content. This may suggest that below $8 \mathrm{wt} \%$ the nanotubes were not physically in contact with each other and conduction mechanism took place through a tunneling process across polymer layers rather than through classical percolation network 
which involved a direct contact between carbon nanotubes [35]. It may also indicate that the tunneling distances between nanotubes in the nanocomposite prepared by SC2 were uniform and the network structure produced by individually dispersed nanotubes more prevalence than the network of agglomerated nanotubes. The above results are consistent with the previous studies on PP/MCNT composite [43] and suggest that using of high shear during compounding favors the formation of conduction paths in the nanocomposite and decrease the percolation threshold.

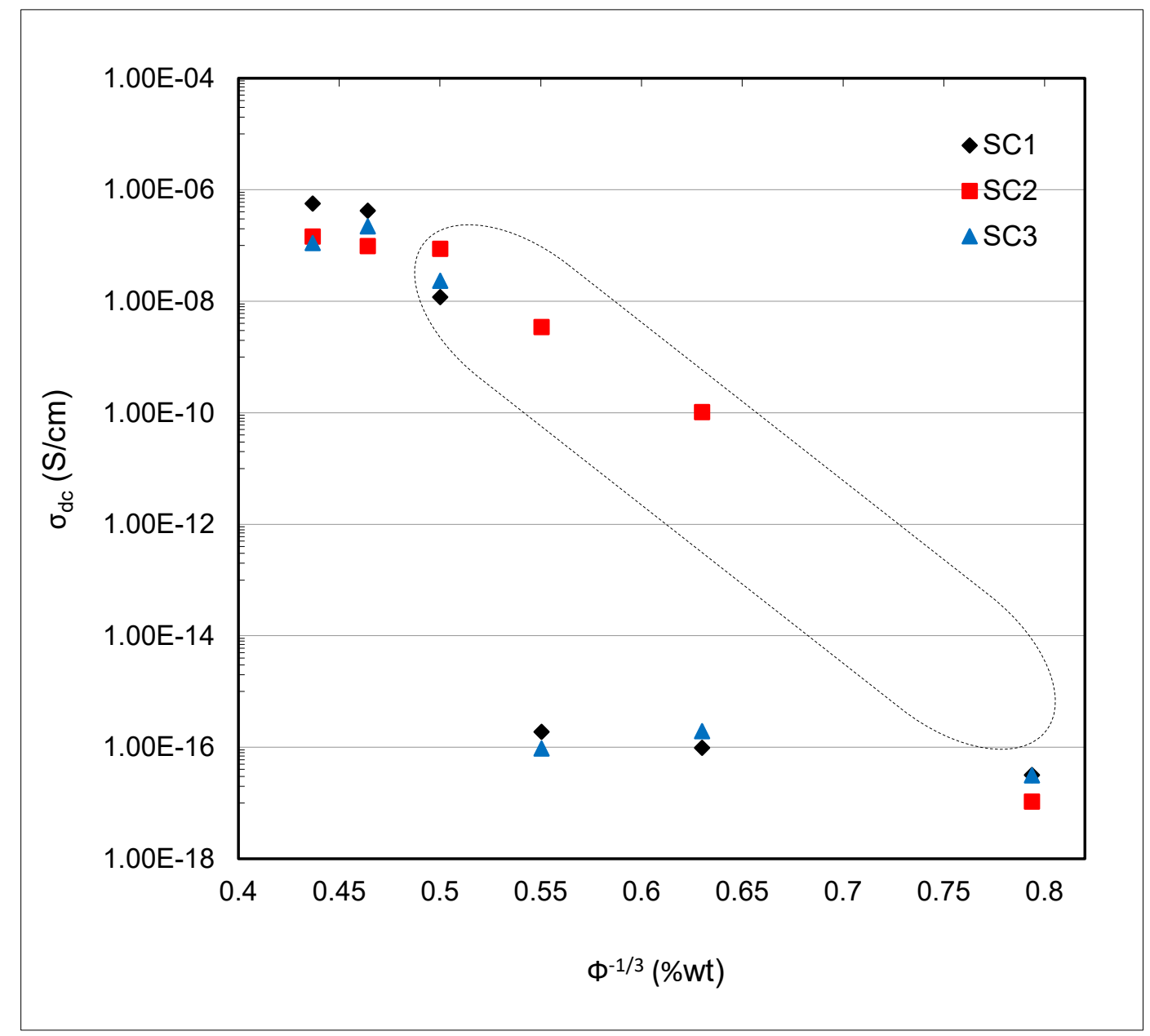

Figure 8 Logarithmic plot of conductivity versus $\Phi^{-1 / 3}$ for nanocomposites prepared by screw configurations $\mathrm{SC} 1, \mathrm{SC} 2$ and $\mathrm{SC} 3$. 


\subsection{Characterization of MCNT Dispersion}

Figure 9 shows SEM images of PP/4wt $\%$ MA2 containing $6 \mathrm{wt} \%$ of nanotubes prepared using the screw configurations (SC1-SC3). Differences in the morphology of the nanocomposites prepared with different screw configurations can be observed. Figure 9a shows that the dispersion of carbon nanotubes in the nanocomposite prepared with the medium intensity screw configurations (SC1) was relatively homogeneous and only small aggregations of about $2 \mu \mathrm{m}$ diameter were detected. The white bright dots appeared in the micrographs were attributed to homogenously dispersed nanotube stubs. The nanocomposite prepared by the high intensity screw configuration ( $\mathrm{SC} 2$ in Figure $9 \mathrm{~b}$ ) was almost free of agglomerates and the nanotubes appeared to be dispersed homogeneously at the micro scale. Large agglomerations of about $30 \mu \mathrm{m}$ were observed for the nanocomposite prepared with the chaotic screw configuration (SC3 in Figure 9c). This was possibly caused by the lower levels of shear imparted by the folding elements compared to mixing paddles, or by re-agglomeration of nanotubes in the slower flow regime. The highest level of nanotube dispersion was observed in the nanocomposite prepared with SC2 which may be due to the presence of large number of kneading elements and back-conveying elements resulting in generation of high stresses which overcame the strong Van der Waals interaction between the nanotubes. These observations are consistent with the electrical resistivity measurements and may explain that the lower electrical percolation in the nanocomposite prepared with screw configuration SC2 was due to the homogeneous dispersion of nanotubes. 

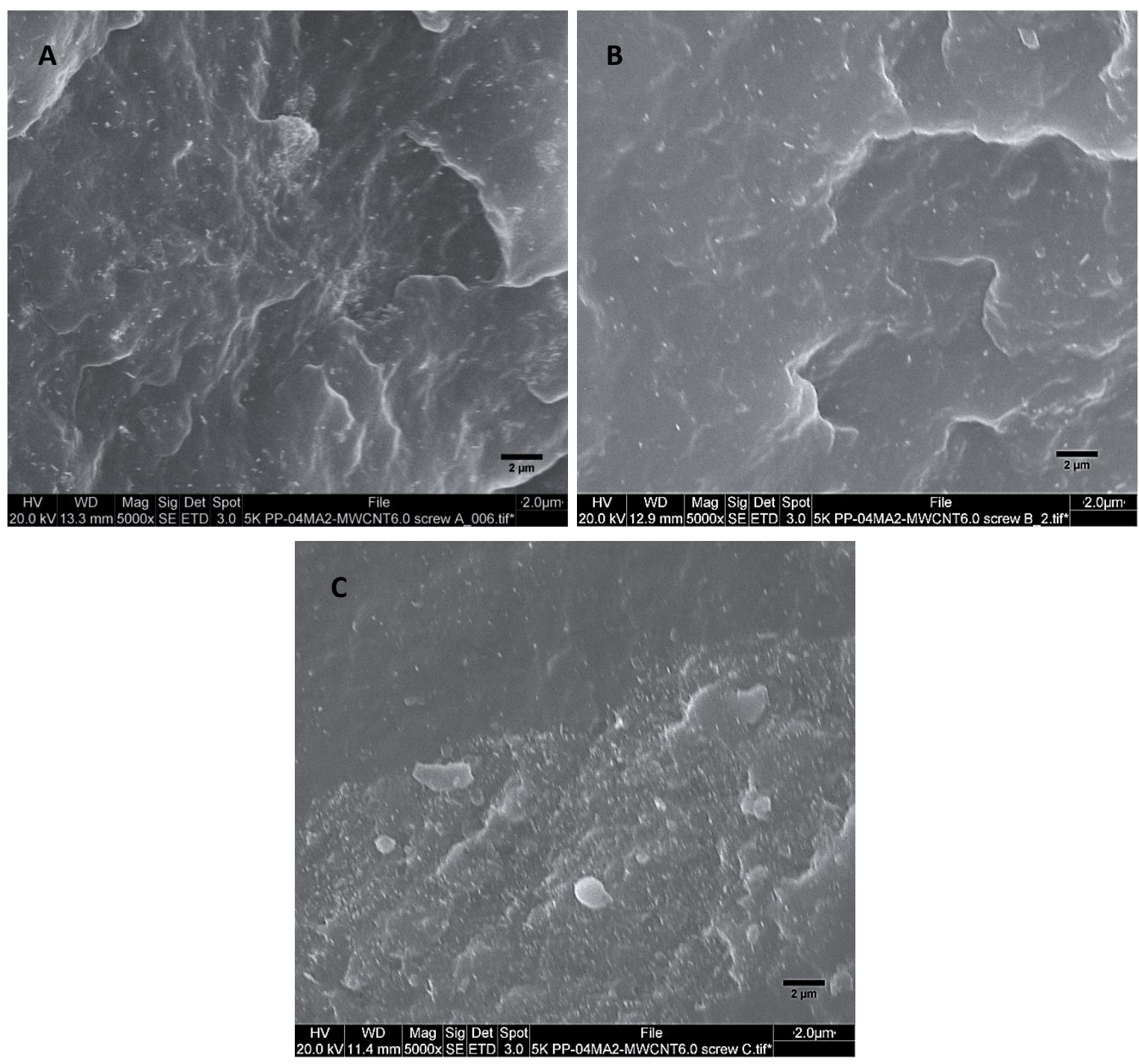

Figure 9 SEM images of compatibilised polypropylene containing $6 \mathrm{wt} \% \mathrm{MCNT}$ prepared by (A)SC1 (B)SC2 and (C) SC3 screw configurations 


\subsection{Residence Time Distribution}

Since mixing time is one of the key parameters affecting the dispersion of carbon nanotubes in polymer nanocomposite, it is important to determine the influence of screw configuration on the mean residence time. Table 2 represents feeding rates and the calculated values of mean residence time measurements for $\mathrm{SC} 1, \mathrm{SC} 2$ and $\mathrm{SC} 3$. From Table 2 it can be seen that the longest residence time was observed in experiments performed using the chaotic screw configuration (SC3). This is possibly due to predominantly non conveying behavior of the folding elements which caused a more intensive recirculation in the extruder. Due to the presence of back-conveying elements, the high intensity screw configuration (SC2) showed $+89 \%$ longer residence time than the medium intensity screw (SC1). A previous investigation found that the value of residence time exponentially decreased with increasing feeding rates, regardless to the type of screw configuration [25]. Therefore, another possible reason for detecting the longest value of residence time in SC3 is due to the slower feeding rates used for SC3 than SC2 and SC1. It is interesting to note that the screws consisting of kneading elements exhibited shorter residence time than the screw consisting of folding elements, the use of this screw led to significantly better nanotube dispersion.

\begin{tabular}{|c|c|c|}
\hline Screw configurations & Mean residence time (S) & Feeding rates (Kg/hr) \\
\hline SC1 & 94 & 900 \\
\hline SC2 & 179 & 600 \\
\hline SC3 & 236 & 500 \\
\hline
\end{tabular}

Table 2 Values of mean residence time and feeding rate for screw configurations SC1, SC2 and SC3. 


\section{Conclusions}

The effect of extruder screw configuration on the tensile and electrical properties of compatibilised polypropylene/multi-walled carbon nanotube composites was studied. Three screw configurations with medium, chaotic and high mixing intensity were designed based on changing the type and position of screw elements in the mixing and melting zones. Extruder screw configurations were found to have a marginal effect on the tensile and rheological properties of PP/MCNT composite. The tensile and rheological properties of the nanocomposite prepared by a screw with medium intensity were found to be the greatest. This was explained by the competition between dispersion and degradation of polymer chains and a possible reduction of nanotube length by intensive shear experience during compounding. The most significant effect of screw configuration was observed in electrical resistivity measurements. The use of a screw configuration consisted of kneading elements and a reverse conveying element (to promote high intensity mixing) was found to be beneficial in decreasing the electrical percolation threshold from $6 \mathrm{wt} \%$ nanotube contents to $2 \mathrm{wt} \%$. Morphological examination indicated the highest level of carbon nanotube dispersion was achieved in the nanocomposite prepared by a screw consisted of kneading elements and a reverse conveying element. This was attributed to the presence of the reverse conveying elements in the screw and the higher mixing intensity of the kneading elements relative to the folding or conveying elements. Overall, the results indicated that the selection of screw element was an important factor to control dispersion and that screw configuration could be tailored to improve specific properties of the nanocomposites. 


\section{References}

1- GS Ezat, The influence of multi-walled carbon nanotubes on the properties of polypropylene nanocomposite, Doctor of Philosophy thesis, University of Bradford, 2012.

2- F Vahedi, HR Shahverdi, MM Shokrieh, M Esmkhani, Effects of carbon nanotube content on the mechanical and electrical properties of epoxy-based composites, Carbon, New Carbon Materials, 29 (2014) 419-425.

3- XC Guang, L Yongjin and S Hiroshi, Ultrahigh-shear processing for the preparation of polymer/carbon nanotube composites, Carbon, 45 (2007) 2334-2340.

4- WK Park, JH Kim, SS Lee, J Kim, GW Lee and M Park, Effect of carbon nanotube pretreatment on dispersion and electrical properties of melt mixed multi- walled carbon nanotubes/poly(methyl methacrylate) composites, Macromol. Res. 13 (2005) 206-211.

5- I Alig, P Pötschke, D Lellinger, T Skipa, S Pegel, GR Kasaliwal and T Villmow, Establishment, morphology and properties of carbon nanotube networks in polymer melts, Polymer, 53 (2012): 4-28.

6- O Zetina-Hernández, S Duarte-Aranda, A May-Pat, G Canché-Escamilla, J UribeCalderon, Coupled electro-mechanical properties of multiwall carbon nanotube/polypropylene composites for strain sensing applications, J. Mat. Sci. 48 (2013) 7587-7593.

7- A Rahmatpour and A Jamal, Steady shear rheological behavior, mechanical properties, and morphology of the polypropylene/carbon nanotube nanocomposites, J. Macromol. Sci. B, 47 (2008) 929-941 .

8- J-M Thomassin, I Huynen, R Jerome, C Detrembleur, Functionalized polypropylenes as efficient dispersing agents for carbon nanotubes in a polypropylene matrix; application to electromagnetic interference (EMI) absorber materials, Polymer, 51 (2010) 115-121. 
9- Y Liu, J Zuo, J Qin and C Li, Structure and tensile properties of polypropylene/carbon nanotubes composites prepared by melt extrusion, Mater Sci-Poland J. 32 (2014) 442447.

10-G Ezat, A Kelly, S Mitchell, M Youseffi, PD Coates, Influence of maleic anhydride compatibiliser on properties of polpropylene/multiwalled carbon nanotube composites, Plast Rbber Compos. 40 (2011) 438-448.

11-AA Koval'chuk, VG Shevchenko, AN Shchegolikhin, PM Nedorezova, AN Klyamkina and AM Aladyshev, Effect of carbon nanotube functionalization on the structural and mechanical properties of polypropylene/MWCNT composites, Macromol. 41(2008) 7536-7542.

12- A Funck, W Kaminsky, Polypropylene carbon nanotube composites by in situ polymerization. Comp. Sci. Technol. 67 (2007) 906-915.

13-TE Chang, LR Jensen, A Kisliuk, RB Pipes, R Pyrz and AP Sokolov, Microscopic mechanism of reinforcement in single-wall carbon nanotube/polypropylene nanocomposite, Polym J. 46 (2005)439-444.

14-BP Grady, F Pompeo, RL Shambaugh, and DE Resasco, Nucleation of polypropylene crystallization by single-walled carbon nanotubes, J. Phys. Chem. B. 106 (2002) 58525858.

15-C Rauwendaal, Understanding extrusion, Hanser Gardner, Munich, 1998.

16- N Wilkinson and AJ Ryan, Polymer processing and structure development, Kluwer Academic Publishers, Dordrecht, Netherlands, 1998.

17- A T Mark and P O, James Processing of polypropylene-clay nanocomposites: Single screw extrusion with in line supercritical carbon dioxide feed versus twin screw extrusion, J. Appl. Polym. Sci. 103 (2007) 884 - 892. 
18- L Delva, T Van De Keere, R Alves, K Ragaert, A Gaspar-Cunha, L Cardon and J Degrieck, Extrusion and characterization of nanoclay filled polypropylene, APEM journal. 8 (2013) 88-95.

19-Z Zhou, S Wang, L Lu, Y Zhang and Y Zhang, Functionalisation of multi- wall carbon nanotubes with silane and its reinforcement on polypropylene composites, Compos. Sci. Technol. 68 (2008) 1727-1733.

20-F Aviles, JV Cauich-Rodrígueza, L Moo-Taha, A May-Pata and R Vargas-Coronado, Evaluation of mild acid oxidation treatments for MWCNT functionalisation, Carbon, 47 (2009) 2970-2975.

21-GS Ezat, AL Kelly, SC Mitchell, M Youseffi. And PD Coates, Effect of maleic anhydride grafted polypropylene compatibilizer on the morphology and properties of polypropylene/multiwalled carbon nanotube composite, Polym. Compos. 33 (2012) 1376-1386.

22-B Mayoral, G Garrett and T McNally, Influence of screw profile employed during melt mixing on the micro-scale dispersion of MWCNTs in poly(propylene), Macromol. Mater. Eng. 299 (2014) 748-756.

23- Jing Zhong, Avraam I. Isayev, Ultrasonic Treatment of PP/CNT Composites During Twin-Screw Extrusion: Effect of Screw Configuration, Polym. Compos. 38 (2017) 26952706.

24-T Villmow, P Pötschke, S Pegel, L Häussler and B Kretzschmar, Influence of twin-screw extrusion conditions on the dispersion of multi-walled carbon nanotubes in a poly(lactic acid) matrix, Polymer, 49 (2008) 3500-3509.

25-T Villmow, B Kretzschmar and P Pötschke, Influence of screw configuration, residence time, and specific mechanical energy in twin-screw extrusion of polycaprolactone/multiwalled carbon nanotube composites, Compos. Sci. Technol. 70 (2010) 2045-2055. 
26- NJ Ward, HGM Edwards, AF Johnson, DJ Fleming and PD Coates, Application of raman spectroscopy for determining residence time distributions in extruder reactors, Appl. Spect. , 50 (1996) 812-815.

27- $\mathrm{R}$ Andrews, D Jacques, $\mathrm{M}$ Minot and $\mathrm{T}$ Rantell, Fabrication of carbon multiwall nanotube/polymer composites by shear mixing, Macromol. Mater. Eng. 287 (2002) 395403.

28-DL Gao and MS Zhan, Fabrication and electrical properties of CNT/PP conductive composites with low percolation threshold by solid state alloying, Polym. Compos. 31 (2009) 1084 - 1090.

29- SY Fu, ZK Chen, S Hong and CC Han, The reduction of carbon nanotube (CNT) length during the manufacture of $\mathrm{CNT} /$ polymer composites and a method to simultaneously determine the resulting CNT and interfacial strengths, Carbon, 47 (2009) 3192-3200

30-K Prashantha, J Soulestin, M F Lacrampe, M Claes, G Dupin and P Krawczak, Multiwalled carbon nanotube filled polypropylene nanocomposites based on masterbatch route: Improvement of dispersion and mechanical properties through PP-g- MA addition, Express. Polym. Lett. 2 (2008) 735-745.

31- T McNally, P Pötschke, P Halley, M Murphy, D Martin, SEJ Bell, GP Brennan, D Bein, $\mathrm{P}$ Lemoine and JP Quinn, Polyethylene multiwalled carbon nanotube composites, Polymer, 46 (2005) 8222-8232.

32-KQ Xiao, LC Zhang and I Zarudi, Mechanical and rheological properties of carbon nanotube-reinforced polyethylene composites, Compos. Sci. Technol. 67 (2007) 177-182.

33- JY Kim and SH Kim, Influence of multiwall carbon nanotube on physical properties of Poly(ethylene 2,6-naphthalate) nanocomposites, J. Polym. Sci. Part B: Polym. Phys. 44 (2006) 1062-1071.

34-S Abbasi, A Derdouri and P J Carreau, Properties of microinjection molding of polymer multiwalled carbon nanotube conducting composites, Polym. Eng. Sci. 51, (2011), 9921003. 
35- W Bauhofer and JZ Kovacs, A review and analysis of electrical percolation in carbon nanotube polymer composites, Compos. Sci. Technol. 69 (2009) 1486-1498.

36- P Pötschke, SM Dudkin and I Alig, Dielectric spectroscopy on melt processed polycarbonate-multiwalled carbon nanotube composites, Polymer, 44 (2003) 5023-5030.

37- Y Zeng, P Liu, J Du, L Zhao, PM Ajayan and H-M Cheng, Increasing the electrical conductivity of carbon nanotube/polymer composites by using weak nanotube-polymer interactions, Carbon, 48 (2010) 3551-3558.

38-JZ Kovacs, BS Velagala, K Schulte and W Bauhofer, Two percolation thresholds in carbon nanotube epoxy composites, Compos. Sci. Technol. 67 (2007) 922-928.

39- BE Kilbride, JN Coleman, J Fraysse, P Fournet, M Cadek, A Drury, S Hutzler, S Roth and WJ Blau, Experimental observation of scaling laws for alternating current and direct current conductivity in polymer-carbon nanotube composite thin films, J. Appl. Phys. 92 (2002) 4024-4030.

40-MO Lisunova, YP Mamunya, NI Lebovka and AV Melezhyk, Percolation behaviour of ultrahigh molecular weight polyethylene/multi-walled carbon nanotubes composites, Eur. Polym. J. 43 (2007) 949-958.

41-V Antonucci, G Faiella, M Giordano, L Nicolais and G Pepe, Electrical properties of single walled carbon nanotube reinforced polystyrene composites, Macromol Symp. 247 (2007) 172-181.

42-DK Chakravarthi, Carbon nanotubes filled polymer composites: A comprehensive study on improving dispersion, network formation and electrical conductivity, Doctor of Philosophy thesis, Rice University, 2010.

43-SC Tjong, GD LIiang and SP Bao, Electrical behavior of polypropylene/multiwalled carbon nanotube nanocomposites with low percolation threshold, Scr Mater. 57 (2007) 461-464. 